\title{
Auditory adaptation to gradual rise or fall in intensity of a tone
}

\author{
ANTONY REINHARDT-RUTLAND \\ Ulster College, Northern Ireland Polytechnic, Newtonabbey, County Antrim, Northern Ireland
}

and

\author{
STUART ANSTIS \\ York University, Downsview, Ontario M3J 1P3, Canada
}

\begin{abstract}
The auditory system adapts to gradual changes of intensity. After listening to a gradually loudening (or softening) tone, a steady test tone appeared to be growing gradually softer (or louder). "Tuning curves" for these aftereffects were measured by adapting to one audiofrequency and testing at another. Softening tones produced larger aftereffects, which were more sharply tuned for audiofrequency, than did loudening tones.
\end{abstract}

Research in vision has profited greatly from parallel investigations of visual coding and of visual perception. Direct physiological measurements of single neural units and psychophysical measurements of visual adaptation and aftereffects have converged upon the notion of visual channels that are tuned to respond to specific features of the visual world (reviewed by Anstis, 1975; Over, 1971). The same strategy has proved increasingly successful in auditory research.

Physiological studies have found neurons responding to frequency modulation (Whitfield \& Evans, 1965), clicks (Gersuni \& Vartarian, 1973), rise times (Suga, 1971), and movement of sound sources (Altman, 1968; Altman, Syka, \& Shmigidina, 1970). Correspondingly, perceptual adaptation effects have been reported from sinusoidal frequency modulation (Kay \& Matthews, 1972) and also from listening to repeated speech consonant sounds (Cooper, 1975). Grantham and Wightman (1979) have reported auditory motion aftereffects: following exposure to a sound source that moved from left to right, a stationary test sound source appeared to be moving from right to left. Anstis and Cavanagh (1979) reported a sensorimotor adaptation to frequency-shifted auditory feedback, which is an auditory analog of visual adaptation to prisms.

Reinhardt-Rutland (1980) and Reinhardt-Rutland and Anstis (1977) have described an auditory aftereffect produced by a gradual change of amplitude. After adaptation to a gradually loudening (or softening) tone, a steady test tone appeared to be growing

This paper is an expanded version of a summary given by Reinhardt-Rutland and Anstis (1977). The research was supported by Grant A 0260 from the Natural Science and Engineering Council of Canada (NSERC). gradually softer (or louder). This is analogous to a visual adaptation to gradual luminance change (Anstis, 1967). The present paper measures two properties of this auditory aftereffect: its dynamic range and its audiofrequency "tuning curve."

\section{METHOD}

Subjects listened for 2 min to an adapting tone of audiofrequency $500 \mathrm{~Hz}, 1 \mathrm{kHz}$, or $2 \mathrm{kHz}$, which was amplitude-modulated by a logarithmic ramp waveform (Figure 1). To avoid extremes of intensity, the adapting ramp was made repetitive. The depth of modulation of the adapting tone was $36 \mathrm{~dB}$, the ramp repetition rate was $.25 \mathrm{~Hz}$, and its rate of amplitude change was $\pm 9 \mathrm{~dB} / \mathrm{sec}$. The rapid rise/fall times of the ramp waveforms lasted about $.2 \mathrm{msec}$; they were perceived as atonal clicks and apparently did not contribute to the effects. The mean level of the adapting tone and the onset of the test tone were at a comfortable listening level of about $\mathbf{4 0} \mathrm{dB}$ SPL. The aftereffect was a continuous (nonrepetitive) increase in subjective loudness. Test tones were then presented for $2 \mathrm{sec}$, interspersed with $10-\mathrm{sec}$ readaptation periods. The test tone was not steady in amplitude but had an adjustable logarithmic ramp. The subject was instructed to adjust the gradual rise or fall time of the test tone's amplitude until it sounded apparently steady, thus nulling the aftereffect. His null setting, which gave a measure of the initial strength of the aftereffect, was recorded.

\section{Experiment 1: Asymmetry Between Loudening and Softening-Effects of Dynamic Range}

Reinhardt-Rutland (1980) found that aftereffects from softening tones were larger than those from loudening tones. He measured aftereffects from amplitude ramps that were fixed at $\pm 18 \mathrm{~dB} / \mathrm{sec}$, over a wide range of audiofrequencies from $125 \mathrm{~Hz}$ to $4 \mathrm{kHz}$. The mean aftereffects following adaptation to softening tones were 4.9 and $3.3 \mathrm{~dB} / \mathrm{sec}$ for his two practiced subjects, but, following adaptation to loudening tones, they were only 2.0 and $2.5 \mathrm{~dB} / \mathrm{sec}$. We have confirmed that this asymmetry still existed when 


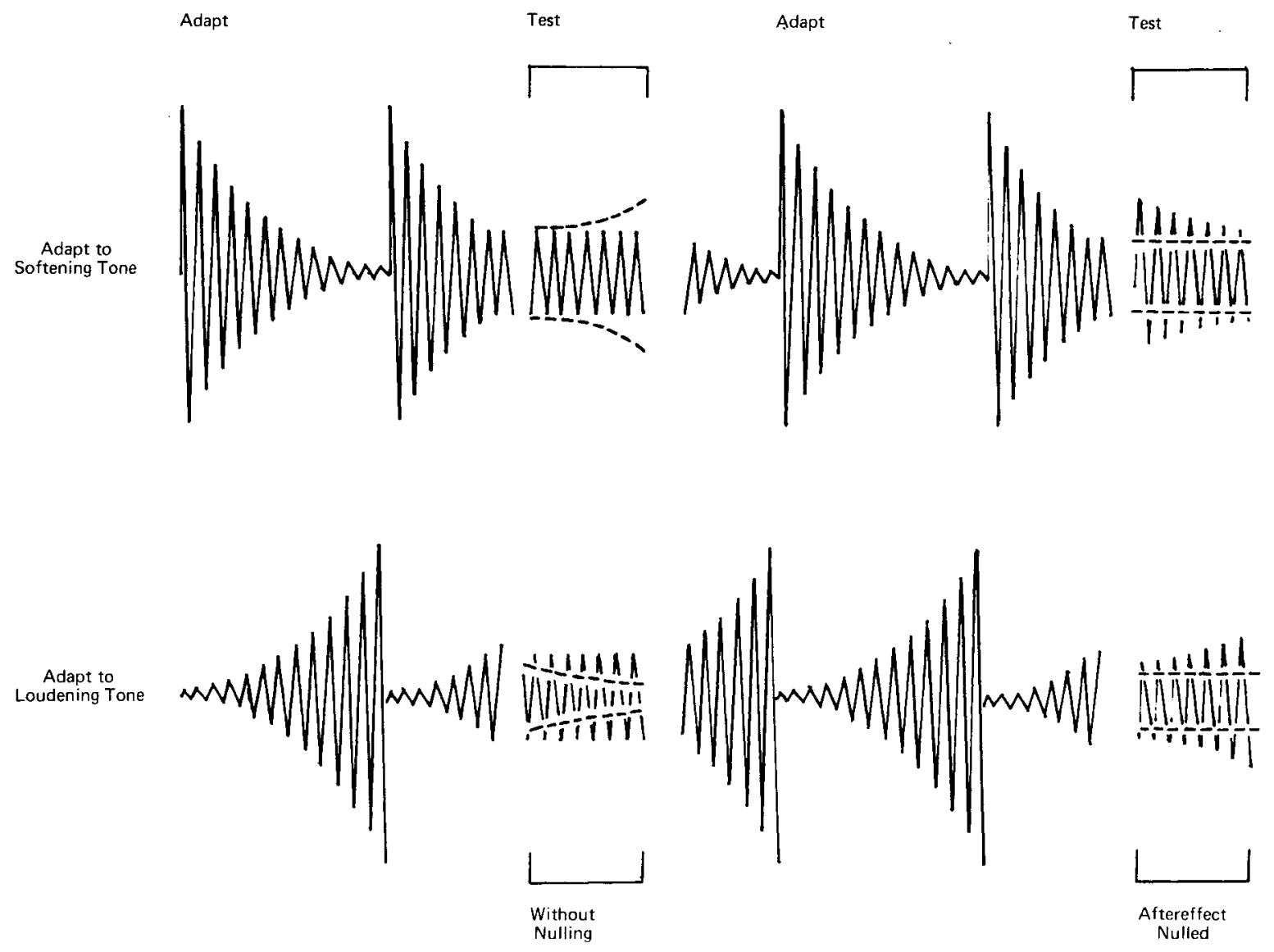

Figure 1. Schematic diagram of the adaptation-test sequence. Broken lines indicate the apparent change in loudness. Subjects adapted for $2 \mathrm{~min}$ to a tone whose sound level was amplitude-modulated by a ramp. A sequence of test tones and readapting stimuli was then presented. In this case, the adapting stimulus was decreasing in amplitude, and the test tone on the left was steady in amplitude and was heard as gradually increasing in loudness. The test tone on the right is set so that it was heard as approximately steady. Onset and offset of test tones were signaled by clicks. Readaptation stimuli always commenced at the same phase of ramp modulation.

we varied the dynamic range of the adapting ramp.

The dynamic range, or depth of modulation, of the adapting amplitude sweep was varied over a range from 3.75 to $9 \mathrm{~dB} / \mathrm{sec}$. (Ramp repetition rate was held constant at $.25 \mathrm{~Hz}$.) This affected the strength of the aftereffects, expressed in $\mathrm{dB} / \mathrm{sec}$ of subjective loudness change. Figure 2 shows that, over the range studied, the greater was the depth of adapting modulation, the greater the subjective aftereffect. Moreover, aftereffects from softening adapting tones were consistently larger than aftereffects from loudening tones: Adaptation to a 9-dB/sec ramp gave an aftereffect of about $3 \mathrm{~dB} / \mathrm{sec}$ following softening tones, but only about $2 \mathrm{~dB} / \mathrm{sec}$ following loudening tones. In consequence, aftereffects from softening were more sensitive to changes in the depth of adapting modulation (steeper slope in Figure 2). Each 1-dB/sec increase in the rate of adapting sound-level change produced an average increase of $.37 \mathrm{~dB} / \mathrm{sec}$ in the aftereffect from softening tones, but of only $.22 \mathrm{~dB} /$ sec in the aftereffect from loudening tones.
These results may reflect a more general asymmetry between the perception of increasing vs. decreasing sound amplitude. There is a tendency to hear small decreases in sound level-but not small increases-as steady (Rawdon-Smith \& Grindley, 1935). Also, naive subjects who are required to make a forced-choice response of "growing louder" or "growing softer" to a series of short steady tones generally make more "growing louder" responses (Small, 1977).

\section{Experiment 2: Audiofrequency Tuning Curves}

Auditory adaptation effects are rather dependent on matching of the audiofrequency in adapting and test stimuli (Cutting, Rosner, \& Foard, 1976; Bailey, Note 1). In our next experiment, the adapting modulation rate was held constant at $\pm 9 \mathrm{~dB} / \mathrm{sec}$ and "tuning curves" for the aftereffects were measured by adapting at one audiofrequency and testing at another. The test audiofrequency was held constant, while the adapting audiofrequency was randomly set to a series 


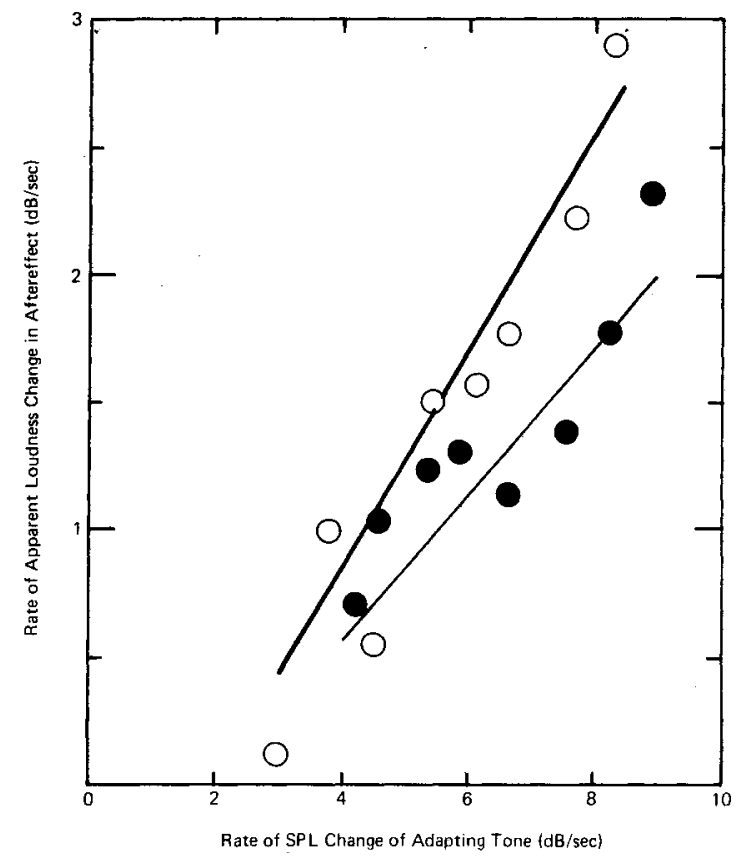

Figure 2. Dynamic range plots for aftereffects. Abscissa $=$ rate of sound level change of adapting tone $(\mathrm{dB} / \mathrm{sec})$. Ordinate $=$ rate of subjective loudness change in aftereffect $(\mathrm{dB} / \mathrm{sec})$. Unfilled circles and thick lines are for adaptation to swept decrease in sound level (softening tones). Filled circles and thin lines are for adaptation to swept increase in sound level (loudening tones). Mean slope was .37 for thick lines but only .22 for thin lines, showing that aftereffects for softening tones increased more steeply with increasing depth of adapting modulation.

of values on different sessions. Five null settings of the test tone were made at each adapting frequency. and $5 \mathrm{~min}$ were allowed for deadaptation before adaptation was begun to a different frequency. Aftereffects from loudening and from softening tones were measured on different days. Subjects also made baseline null settings of test stimuli without any adaptation stimulus. Plus or minus 1 SD of these data (shown as broken horizontal lines in Figure 3) were arbitrarily taken as baselines against which adaptation effects could be judged. The test audiofrequencies were 1 and $2 \mathrm{kHz}$ for one subject and 500,1 , and $2 \mathrm{kHz}$ for the other subject.

Results are shown in Figure 3. Note the asymmetries between aftereffects from softening tones (upper half of Figure 3) and from loudening tones (lower half). Although tuning curves showed differences between subjects, the peak aftereffects were 1.23 times greater for A.H.R.R. and 1.11 times greater for V.L. following softening tones than following loudening tones. Also, the aftereffects following softening were more sharply tuned than following loudening: the mean half bandwidths were .83 (A.R.) and 1.25 (V.L.) octaves from a softening tone and 1.25 (A.R.) and 2.45 (V.L.) octaves from a loudening tone. These represent bandwidth ratios of 1.50 for A.R. and 1.96 for V.L.

Thus, loudening and softening tones produced asymmetrical types of aftereffects. In addition to

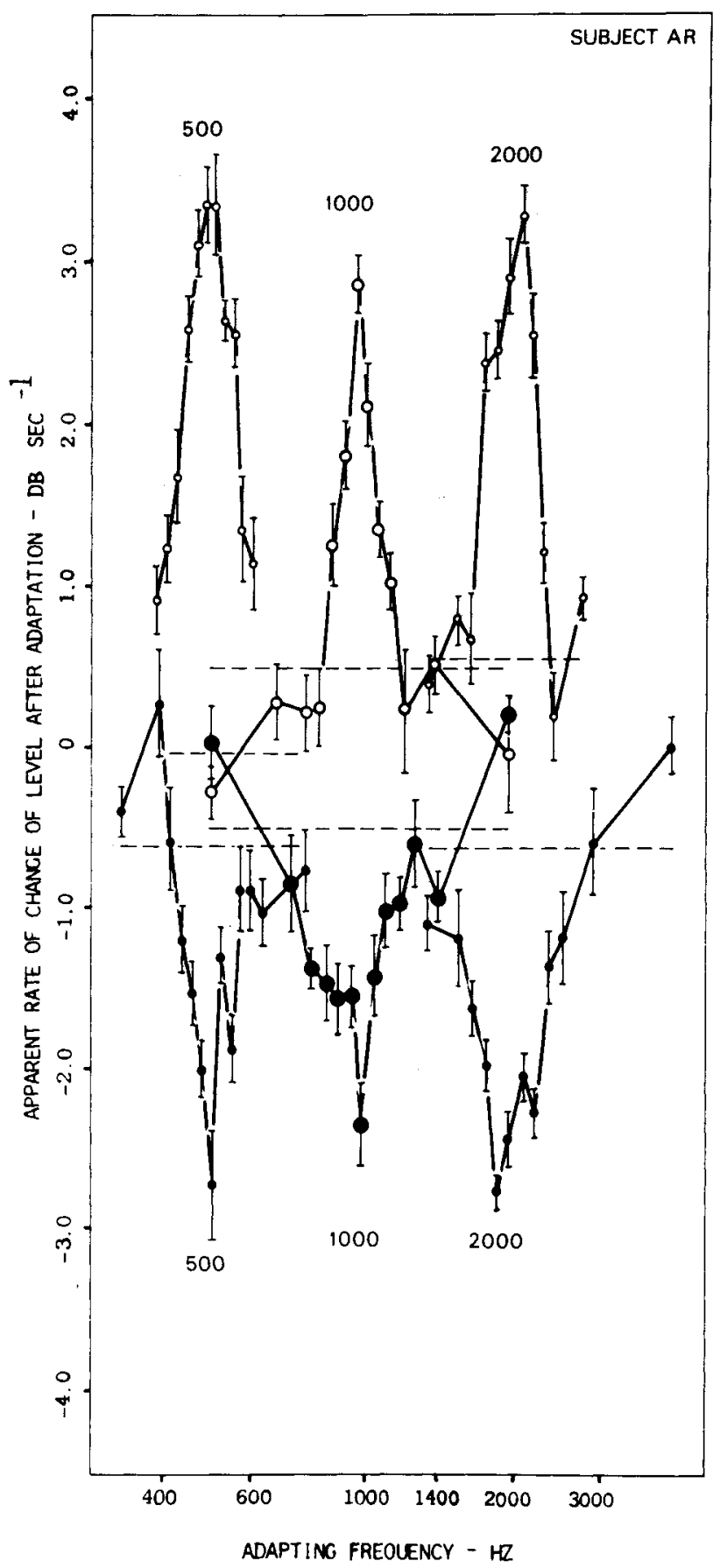

Figure 3. Audiofrequency tuning curves for aftereffects (Subject A.R.) Filled (unfilled) circles are for adaptation to swept increase (decrease) of sound pressure level. Vertical bars are \pm 1 SD. Broken horizontal lines are baselines. The test frequency for each function is shown by a figure above or below each tuning curve. 
producing larger aftereffects, softening tones were much more sharply tuned for audiofrequency.

\section{DISCUSSION}

Neurons have been found in the inferior colliculus that are sensitive to swept amplitude change (Cooper, 1975; Kay \& Matthews, 1972; Nelson, Erulkar, \& Bryan, 1966). These aftereffects may indicate adaptation in such neurons.

The fact that aftereffects are greater from softening than from loudening tones suggests slight differences in the corresponding neural channels. These differences may reflect the time asymmetry of many natural sounds, which, being percussive, have short rise times and relatively long decay times. An amusing way to appreciate this is to listen to tape recordings of various sounds played backward. Music contains many percussive sounds, and a piano played backward sounds like an organ, while drum beats sound very strange. Speech sounds are less asymmetrical: English speech played backward does not sound like English, but it still sounds like a language. Probably the biggest time asymmetries are found in echoes, which are always fainter than the primary sound and have relatively long decay times. The auditory system is good at suppressing or ignoring echoes. Echoes may pass unnoticed on a tape recording of sounds made in an echo chamber, but when the tape is played backward the echoes precede the original sounds and are extremely intrusive. It is at least possible that the different responses that we found to loudening and softening tones may be related to the perceptual process of echo suppression.

The tuning curves of our aftereffects had much broader bandwidths than do "critical bands," as reviewed by Scharf (1970). Estimates of critical bandwidths vary somewhat, depending on whether they are measured by techniques of loudness summation, masking, or adaptation. But the most relevant measure for comparison with our aftereffects comes from Thwing's (1955) adaptation technique. He adapted his subjects for $5 \mathrm{~min}$ to an intense steady tone of $1,000 \mathrm{~Hz}$ at $80 \mathrm{~dB}$ SPL and then measured threshold elevation at the same and neighboring audiofrequencies. He found critical bandwidths of only $\pm 150 \mathrm{~Hz}$ (about .25 octaves), compared with our aftereffect bandwidths of between .83 and 2.45 octaves. Clearly, Thwing's experiment and ours were not adapting the same neural channels. We speculate that each of our broadly tuned channels, sensitive to dynamic changes, may be receiving inputs from many of Thwing's narrowly tuned channels. Thus, our channels have good sensitivity to the sound envelope, at the price of poor frequency discrimination. This may be analogous to the visual channels for seeing motion, which have good dynamic response to mov- ing objects at the price of poor spatial acuity. Such motion-sensitive channels are thought to have large receptive fields and to receive inputs from many channels sensitive to static position. Each static channel has a small receptive field and high spatial acuity. Thus, the auditory channels that achieve precise frequency discrimination are probably different from the auditory channels we studied, which respond primarily to amplitude changes in the sound envelope.

\section{REFERENCE NOTE}

1. Bailey, P. J. Preliminaries to an account of formant transition perception (Speech Perception 2.2, Progress Report of the Department of Psychology). Belfast: Queen's University, 1973.

\section{REFERENCES}

Altman, J. A. Are there neurons detecting direction of sound source movement? Experimental Neurology, 1968, 22, 13-25.

Altman, J. A., Syka, J., \& Shmigidina, G. N. Neuronal activity in the medial geniculate body of the cat during monaural and binaural stimulation. Experimental Brain Research, 1970, 10, 81-93.

Anstis, S. M. Visual adaptation to gradual change of intensity. Science, 1967, 155, 710-712.

Anstis, S. M. What does visual perception tell us about visual coding? In M. Gazzaniga \& C. Blakemore (Eds.), Handbook of psychobiology. New York: Academic Press, 1975.

Anstis, S. M., \& Cavanagh, P. Adaptation to frequency-shifted auditory feedback. Perception \& Psychophysics, 1979, 26, 449458.

Cooper, W. E. Selective adaptation to speech. In E. Restle, R. M. Shiffrin, J. N. Castellan, H. Lindman, \& D. B. Pisoni (Eds.), Cognitive theory. Potomac, Md: Erlbaum, 1975.

Cutring, J. E., Rosner, B. S., \& Foard, C. F. Perceptual categories for music like sounds: Implications for theories of speech perception. Quarterly Journal of Experimental Psychology, 1976, 28, 361-379.

Gensuni, G. V., \& Vartanyan, I. A. Time dependent features of adequate sound stimuli and the functional organization of central auditory neurons. In A. R. Moller (Ed.), Basic mechanisms in hearing. New York: Academic Press, 1973.

Grantham, D. W., \& Wightman, F. L. Auditory motion aftereffects. Perception \& Psychophysics, 1979, 26, 403-408.

KAY, R. H., \& MAtThEws, D. R. On the existence in human auditory pathways of channels selectively tuned to the modulation present in auditory frequency-modulated tones. Journal of Physiology, 1972, 225, 657-676.

Nelson, P. G., Erulkar, S. D., \& Bryan, J. S. Responses of units of the inferior colliculus to time-varying acoustic stimuli. Journal of Neurophysiology, 1966, 29, 834-860.

OVER, $R$. Comparison of normalization theory and neural enhancement explanation of negative aftereffects. Psychological Bulletin, 1971, 75, 225-243.

Rawdon-Smith, A. F., \& Grindley, G. C. An illusion in the perception of loudness. British Journal of Psychology, 1935 , 26, 191-195.

Reinhardt-Ruthand, A. H. Two auditory aftereffects and their dependence on carrier frequency. Perception \& Psychophysics, 1980, 28, 569-571.

Reinhardt-Rutrand, A. H., \& Anstis, S. M. Adaptation effects arising from swept amplitude change of tones. Brain Research Association Bulletin, 1977, 25, 8. 
Scharf, B. Critical bands. In J. V. Tobias (Ed.), Foundations of modern auditory theory. New York: Academic Press, 1970.

Small, A. M. Loudness perception of signals of monotonically changing sound pressure. Journal of the Acoustical Society of America, 1977, 61, 1293-1297.

SugA, N. Responses of inferior collicular neurones of bats to tone bursts with different rise times. Journal of Physiology, $1971,217,159-177$.

THWING, E. J. Spread of perstimulatory fatigue of a pure tone to neighboring frequencies. Journal of the Acoustical Society of America, 1955, 27, 741-748.

Whitfield, I. C., \& Evans, E. F. Responses of auditory cortical neurons to stimuli of changing frequency. Journal of Neurophysiology, 1965, 28, 655-672.

(Manuscript received April 9, 1981;

revision accepted for publication October 2, 1981.) 